\title{
Tecnologia na Educação Matemática: Trilhando o Caminho do Fazer ao Compreender
}

\author{
Márcia Rodrigues Notare - IM/PPGEMAT/UFRGS - marcia.notare@ufrgs.br \\ Marcus Vinicius de Azevedo Basso - IM/PPGEMAT/UFRGS - mbasso@ufrgs.br
}

\begin{abstract}
Resumo: Este artigo discute o potencial do software GeoGebra no processo de construção de conceitos matemáticos, à luz da teoria do desenvolvimento cognitivo de Piaget. Para isso, o artigo traz a análise de um projeto de modelagem geométrica, da trajetória de uma bola de basquete, desenvolvido por um aluno do mestrado em Ensino de Matemática, na qual é possível evidenciar um caminho trilhado do fazer ao compreender, na medida em que o trabalho vai sendo desenvolvido. Conceitos matemáticos como função quadrática, função afim, função seno, função valor absoluto, movimentos de gráficos e continuidade de funções foram definitivos na construção do modelo assim como conceitos geométricos como retas paralelas e perpendiculares, ponto médio, mediatriz e reflexão em torno de uma reta, que foram utilizados na construção da cesta. Dessa forma, a atividade proposta com o uso do GeoGebra permitiu a compreensão de uma série de conceitos a partir da ação do sujeito e da conceituação, trilhando um caminho do fazer ao compreender.
\end{abstract}

Palavras-Chave: educação matemática e tecnologia, construção do conhecimento, modelagem geométrica, fazer e compreender.

\section{Technology in Mathematics Education: the Path of Make to Understand}

\begin{abstract}
This article discusses the potential of the software GeoGebra in the construction of mathematical concepts, using Piaget's theory. The article brings the analysis of a geometric modeling project, the trajectory of a basketball, designed by a student of post-graduation in Teaching Mathematics, in which we can show a path trodden of make to understand, insofar that the work is being developed. Mathematical concepts such as quadratic function, affine function, sine function, absolute value function, motion graphics and continuity of functions were definitive in building the model as well as geometric concepts as parallel and perpendicular lines, midpoint, bisector and reflection around a straight, which were used in the construction of the basket. Thus, the proposed activity with the GeoGebra allowed the understanding of concepts from the subject's action and conceptualization, treading a path of make to understand.
\end{abstract}

Keywords: mathematics education and technology, knowledge construction, geometric modeling, make and understand.

\section{Introdução}

Não é de hoje que o processo de aprendizagem de Matemática vem sofrendo fortes críticas por apresentar resultados de um sistema ultrapassado e fracassado. Um dos principais fatores responsáveis por este cenário é que a Matemática ainda é apresentada aos alunos de forma polida, por meio de formalismos organizados em uma sequência de teoremas, demonstrações e aplicações. Dessa forma, omite-se dos alunos o verdadeiro processo de construção dos conceitos envolvidos. A Matemática, ao longo dos tempos, foi desenvolvida por meio de tentativas e erros, a partir de afirmações elaboradas intuitivamente, com imprecisões e afirmações fracas, introduzidas intencionalmente na tentativa de visualizar a estrutura matemática, de forma dinâmica (Tall, 1991). 
Então, porque não apresentá-la sob este enfoque aos alunos? É nesta perspectiva que trazemos uma discussão sobre o uso da tecnologia nas aulas de Matemática. Os softwares disponíveis hoje em dia podem proporcionar um valioso trabalho de construção do conhecimento matemático, desde que as atividades sejam elaboradas com o objetivo de engajar os alunos em um processo de superação de desafios, no qual a utilização de conceitos matemáticos seja necessária para alcançar os objetivos propostos inicialmente.

Dessa forma, buscamos apoio na teoria do desenvolvimento cognitivo de Piaget para evidenciar o potencial de uma atividade de modelagem geométrica com o software GeoGebra. Vamos mostrar, a partir da análise da construção do modelo de um aluno, como essa atividade pode levar à construção de conceitos matemáticos, a partir da ação do estudante, trilhando um caminho do fazer ao compreender.

\section{Aprendizagem de Matemática: trilhando o caminho do fazer ao compreender}

A construção do conhecimento matemático se dá na medida em que novos problemas matemáticos vão sendo vivenciados, uma vez que o aluno é perturbado e desafiado a superá-lo. Para resolver o problema, é preciso que o aluno construa novas estruturas que permitam dar conta da situação enfrentada, rever os conceitos já elaborados e tentar reconstrui-los e enriquecê-los, de forma a solucionar o problema apresentado. Se o aluno não vive o problema como uma contradição interna, não sentirá necessidade de construir algo novo para resolver tal problema. Esse processo é contínuo e são justamente esses desafios que promovem o desenvolvimento do ser humano e, especificamente, a construção do conhecimento matemático.

Dessa forma, à medida que o aluno é desafiado a resolver um novo problema até então desconhecido, um desequilíbrio cognitivo pode desencadear o processo de construção do conhecimento matemático.

Normalmente, o que se observa, é um sucesso aparente dos alunos na resolução de problemas. Isso ocorre porque, geralmente, as aulas de Matemática escolar reforçam a simples utilização e reprodução de procedimentos e algoritmos, enfatizando roteiros ou modelos a serem seguidos na resolução de problemas. Dessa forma, o que ocorre é a aprendizagem de um conjunto de procedimentos padrão, que possibilita a resolução de uma classe de problemas extremamente limitada; os alunos adquirem apenas a capacidade de efetuar cálculos, sem compreendê-los. Esse processo está longe do verdadeiro fazer matemática, que exige habilidades como conjecturar, testar, intuir, deduzir, generalizar - coordenar ações e retirar dessas coordenações novas coordenações, por abstrações refletidas.

\subsection{Tomada de consciência: do fazer ao compreender}

Para compreender melhor como se dá a construção do conhecimento matemático, recorremos à teoria do desenvolvimento cognitivo de Piaget, que trata da origem do conhecimento e o entende como sendo um processo contínuo de construção, sem início ou final absoluto (Piaget, 1983). A inteligência, para Piaget, é construída pelo mecanismo de adaptação do organismo a uma situação nova e, como tal, implica a construção contínua de novas estruturas. Desta forma, os indivíduos desenvolvem-se intelectualmente a partir das vivências e experiências oferecidas pelo meio que os cercam, agindo sobre este meio.

Para Piaget (1983), o processo de construção do conhecimento se constitui na ação do sujeito sobre um objeto (assimilação) e na ação do sujeito sobre si mesmo (acomodação), respondendo às estranhezas trazidas pelo material assimilado. Assim, 
sujeito e objeto não podem ser dissociados, mas entendidos como completares, uma vez que o conhecimento não se encontra pré-existente em nenhum destes polos, mas sim, na sua interação.

Pode-se dizer que o sujeito só aprende porque age. A ação é a força propulsora do desenvolvimento humano, ou seja, é por meio das ações que o sujeito pratica, que ele se desenvolve. Entretanto, não é qualquer ação que leva a avanços no conhecimento; é preciso uma ação significativa, que tenha sentido para o sujeito, que o faça pensar sobre o que fez e sobre o próprio pensamento.

Assim, aprendizagem não significa aprender porque alguém ensina, mas sim, por um processo de construção, de reconstrução e de tomada de consciência do próprio desenvolvimento por parte do sujeito. Nesta perspectiva, tudo acontece pela ação do sujeito, pois é por meio dela que se constroem as estruturas do conhecimento ou capacidades de conhecer.

Nesse processo de desenvolvimento cognitivo, é possível identificar certos níveis, que trilham o caminho do fazer ao compreender. Em outras palavras, pode-se dizer que essa evolução inicia na ação pura, impulsionada pela busca em alcançar determinado objetivo, muitas vezes de fato alcançado, mesmo que sem a compreensão dos meios que levaram a tal êxito, até a total compreensão das ações realizadas. Assim, tal evolução caminha do "fazer" ao "fazer e saber o que se está fazendo".

A ação constitui um conhecimento cuja conceituação somente se efetua por tomadas de consciência posteriores, que procedem da periferia para o centro. Verificase um atraso da conceituação sobre a ação, o que mostra a autonomia da ação. Porém, a partir de determinado nível, há uma influência da conceituação sobre a ação, uma vez que a conceituação fornece à ação um reforço de suas capacidades de previsão, consistindo em um aumento do poder de coordenação, e tornando possível uma programação completa da ação a partir da conceituação. Dessa forma, identifica-se uma fase inicial, em que a ação e sua conceituação são aproximadamente do mesmo nível e em que se efetuam trocas constantes entre as duas, e outra fase em que a conceituação fornece à ação uma programação, fazendo com que a prática se apoie em teorias. Assim, à medida que a compreensão começa a se estabelecer, percebe-se uma capacidade de antecipação e uma regulagem mais ativa, que abre para uma capacidade de escolha entre meios diferentes, sem limitar-se mais às regulações automáticas. Dessa forma, a antecipação e a capacidade de escolha permitem a passagem do nível do comportamento material para o da representação.

Uma vez que a conceituação aumenta o poder de antecipação do sujeito, pode-se dizer que a conceituação influencia a ação, fornecendo à mesma um reforço de suas capacidades de previsão e a possibilidade de dar um plano de utilização imediato. Segundo Piaget (1978), essa contribuição consiste em um aumento do poder de coordenação do sujeito.

O conceito de tomada de consciência é de fundamental importância na aprendizagem de Matemática. Isto porque, muitas vezes, a Matemática é tratada de forma mecânica e repetitiva, fazendo com que os alunos reproduzam leis e fórmulas na resolução de problemas, obtendo êxito na sua solução, mas sem efetivamente compreender o que estão fazendo. $\mathrm{O}$ estudo da tomada de consciência busca aprofundar essas questões, na tentativa de desvendar os verdadeiros limites existentes entre o fazer e o compreender.

Para compreender as razões funcionais da tomada de consciência, deve-se entender que esta se dá por meio de um percurso de um determinado comportamento, que é iniciado com a busca de um fim, onde os dados de observação iniciais são 
denominados de periféricos. Essa periferia não é definida nem pelo objeto, nem pelo sujeito, mas pela reação do sujeito em face do objeto. Dessa forma, Piaget (1977) afirma que a tomada de consciência procede da periferia para o centro, ou seja, dos objetivos e resultados, para o reconhecimento dos meios empregados e das escolhas realizadas, que levaram a um êxito ou fracasso.

Assim, a tomada de consciência consiste em uma conceituação, ou seja, em uma passagem da assimilação prática a uma assimilação por meio de conceitos. Dessa forma, quanto mais o sujeito se limitar às reações elementares, mais ele deformará conceitualmente os dados de observação, levando a leituras deformadas de dados, mesmo em casos de êxito na ação. Essa deformação inferencial não constitui uma característica da tomada de consciência, refletindo a própria inconsciência do sujeito em relação aos meios empregados para obter tal êxito.

Entretanto, nem todo processo de tomada de consciência inicia por tais deformações. Muitas vezes, percebe-se um atraso da conceituação em relação à ação, e não exatamente uma contradição de princípios.

Então, pode-se observar que a tomada de consciência é, desde seu início, um processo de conceituação. As ações do sujeito são vistas por ele e assimiladas adequadamente por sua consciência, tratando-se de uma reconstrução capaz de explicar as conexões observadas no êxito de suas ações.

A tomada de consciência realiza-se a partir dos dados de observação relativos ao objeto; por outro lado, é a análise desses dados de observação que vai fornecer informações sobre o objeto e a explicação causal do seu comportamento. Isso provoca uma ação recíproca dos dados de observação sobre a ação e vice-versa. Ao ser estabelecido um relacionamento entre eles, seguem-se as coordenações inferenciais, que ultrapassam o campo dos dados de observação e permitem ao sujeito compreender causalmente os efeitos observados. Dá-se, então, um processo de idas e vindas, que refina os dados de observação e o grau de compreensão.

\subsection{A teoria de Piaget na aprendizagem de Matemática}

Piaget (1978) afirma que a ação precede a conceituação. Muitas vezes, um sujeito realiza uma determinada ação, impulsionado por determinado objetivo a ser alcançado, e obtêm sucesso em sua realização. Entretanto, não consegue descrever os caminhos que o levaram a esse sucesso, o que mostra a ausência de compreensão dos conceitos envolvidos em seus atos. Situações como essa são frequentemente observadas nas aulas de Matemática. A valorização de métodos e roteiros para a resolução de problemas faz com que alunos resolvam os problemas apresentados sem a necessidade de uma verdadeira compreensão dos conceitos matemáticos envolvidos nesses procedimentos; sem agir sobre as coordenações de suas ações e retirar delas qualidades que lhes são próprias.

No entanto, o verdadeiro trabalho dos matemáticos faz uso de uma experiência que os permite usar o conhecimento matemático de forma flexível, para resolver problemas diferentes e até então desconhecidos. Piaget (1995) afirma que quanto mais o sujeito age sobre o meio, mais ele torna-se capaz de aprender, retirando das coordenações das ações novas coordenações e sintetizando-as em estruturas, como as lógicas e matemáticas. Essa experiência matemática é adquirida por meio de um processo contínuo, a partir de diferentes problemas que permitem novas construções por parte do sujeito.

Dessa forma, acredita-se que quanto mais problemas matemáticos são enfrentados pelo sujeito, quanto mais ele pensar, conjecturar, testar suas hipóteses e 
reorganizar suas ideias, mais experiência matemática ele vai construir, e, consequentemente, mais problemas poderão ser resolvidos por ele, problemas estes até então desconhecidos. Nesse processo, novos conhecimentos vão sendo construídos e a capacidade de resolução de novos problemas vai enriquencendo-se e ampliando-se. Segundo Piaget (1995), a compreensão de uma estrutura torna-se proporcional à extensão dos conteúdos que ela permite engendrar. Em resumo, quanto maior o número de situações novas enfrentadas, mais conhecimento o sujeito constrói e, consequentemente, maior será sua capacidade de revestir os objetos matemáticos de propriedades até então não constatadas, de modo que as abstrações vão tornando-se mais elaboradas.

Entretanto, vale salientar que não se está falando de listas intermináveis de problemas repetitivos, que seguem um mesmo modelo de resolução e não desafiam os alunos nem provocam reflexões e inquietações. Tais problemas devem exigir estratégias diferentes de resolução, bem como diferentes conceitos e propriedades matemáticos.

Os alunos precisam perceber que há um caminho a ser trilhado, que envolve conhecimentos que devem ser construídos por cada sujeito, e que a apresentação de uma solução pronta e enxuta não possibilita vivenciar essa construção.

Por outro lado, sabe-se da importância do raciocínio informal, que permite manipular ideias e imagens mentais na busca de um encaixe que leve a soluções de problemas matemáticos. O ensino da Matemática deve fazer uso da experimentação, da observação e da descoberta. Isso permite uma compreensão em vários estágios necessários ao pensamento matemático, como representação, visualização, generalização, classificação, conjectura, indução, análise, síntese, abstração e formalização (Dreyfus, 1991).

\section{O potencial da tecnologia na aprendizagem da Matemática}

Os recursos tecnológicos disponíveis hoje em dia podem proporcionar um grande avanço no processo de aprendizagem de Matemática. Isso porque temos, atualmente, softwares que vão ao encontro da concepção de construção de conhecimento abordada nesse trabalho.

A partir de atividades elaboradas com suporte na teoria do desenvolvimento cognitivo, é possível lançar aos alunos desafios que provoquem a necessidade de construção de novos conhecimentos, proporcionando o desenvolvimento de habilidades cognitivas que o conduzam do fazer ao compreender.

Um software que apresenta potencial para melhorar a abordagem dada à Matemática nas escolas é o GeoGebra - um software de geometria dinâmica, no qual é possível trabalhar, simultaneamente, com geometria e álgebra Sua interface pode ser visualizada na Figura 1. 


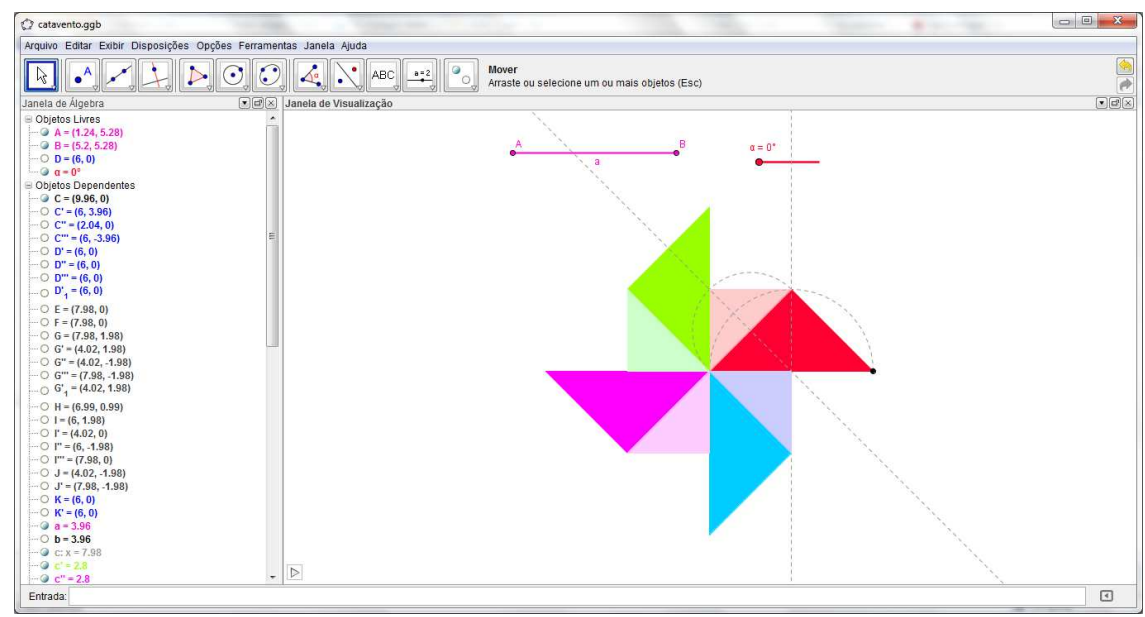

Figura1. Interface do GeoGebra

As construções feitas no GeoGebra são produzidas a partir de menus, que contém ferramentas que permitem construir objetos geométricos, como ponto, reta, polígono, círculo, ângulo, retas paralelas e perpendiculares, entre outros. As figuras devem ser construídas a partir de propriedades que as definem. Isso faz com que estas propriedades sejam mantidas sob a ação do movimento, o que permite a manipulação direta destas figuras pelos alunos para explorar, testar, analisar e conjecturar hipóteses sobre o problema a ser resolvido.

Por outro lado, o software GeoGebra permite uma análise via geometria analítica das figuras construídas, na qual é possível tratar pontos a partir de suas coordenadas no plano, retas e circunferência a partir de suas equações, exibindo o sistema de eixos coordenados e uma janela de álgebra. Este recurso permite que os mesmos objetos matemáticos sejam analisados em suas diferentes representações, o que possibilita uma maior compreensão dos conceitos envolvidos, tanto em extensão quanto em aprofundamento.

Dessa forma, este ambiente torna-se um importante recurso para ser utilizado como um espaço de exploração e manipulação pelos alunos, pois valoriza a ação do aluno, tanto no processo de construção, quanto no processo de exploração. Neste sentido, sua utilização nas aulas de Matemática pode levar os alunos ao processo de tomada de consciência de conceitos matemáticos.

Como já afirmado, a tomada de consciência realiza-se a partir dos dados de observação relativos ao objeto. Mas é a partir da análise desses dados de observação que se obtém informações sobre o objeto e a explicação causal do seu comportamento. Ou seja, o GeoGebra permite o estabelecimento de uma relação entre os dados de observação e a ação do aluno e, nesse processo de idas e vindas, o aluno vai aumentando o grau de compreensão dos conceitos matemáticos envolvidos no problema.

Dessa forma, é possível perceber que a utilização de recursos tecnológicos pode se caracterizar como uma extensão do pensamento do aluno, constituindo um meio para trilhar o caminho do fazer ao compreender.

A seção a seguir apresenta, a partir de um exemplo, o potencial do GeoGebra na compreensão de diversos conceitos matemáticos.

\section{Reflexão sobre uma Experiência}

Em uma disciplina oferecida pelo Programa de Pós-Graduação em Ensino de Matemática, foi lançado o desafio, aos alunos, de realizarem a construção de um modelo 
geométrico que representasse um movimento real. Conforme Gravina and Contiero (2011, p.4), "um modelo matemático é uma representação, na linguagem da matemática, de certos aspectos de um fenômeno e tem por fim trazer um maior entendimento do fenômeno". Nosso objetivo inicial era de que o modelo fosse elaborado do ponto de vista geométrico. Contudo, as produções dos alunos levaram-nos a perceber a riqueza de um software que possibilita o trabalho com geometria e álgebra e o seu potencial na construção do conhecimento matemático.

Para ilustrar este potencial, vamos analisar o trabalho de um dos alunos da disciplina, Wagner, no qual identificaremos por W. O aluno W sentiu-se desafiado em modelar o movimento de uma bola de basquete sendo arremessada.

Foi possível observar, como uma das primeiras atitudes de $\mathrm{W}$, a vontade de desistir, por estar frente a um novo problema, ainda desconhecido. Entretanto, em seguida esse sentimento foi substituído pela necessidade de superar o desafio que o estava perturbando. Foi possível perceber que $\mathrm{W}$ vivenciou o problema como uma contradição interna, que precisava ser superada. Como já afirmado, se o aluno não vive o problema como uma contradição interna, não sentirá necessidade de construir algo novo para resolver o problema. Esse processo é contínuo e são justamente esses desafios que promovem o desenvolvimento do ser humano.

É importante salientar que não houve preocupação em construir um modelo fiel à realidade, mas sim um modelo que desse uma ideia da trajetória da bola. A partir das ideias iniciais de $\mathrm{W}$, vamos discutir o processo de construção deste modelo, para mostrar o quanto uma atividade de modelagem, que faz com que o aluno se engaje na solução do problema, desencadeia um valioso processo de tomada de consciência de conceitos matemáticos.

A primeira hipótese de $\mathrm{W}$ foi de que a trajetória inicial da bola, ao ser arremessada, teria um comportamento parabólico. Neste momento, percebe-se a necessidade de utilizar uma relação matemática que represente o movimento esperado. Para identificar a relação matemática mais adequada, Wagner inicia seu processo na ação pura, escrevendo a conhecida relação $y=x^{2}$. Imediatamente, $\mathrm{W}$ percebe que alguns movimentos de gráfico precisam ser aplicados à relação inicial, para que a parábola posicione-se com a concavidade para baixo, além de translações verticais, horizontais e compressões. Após algumas análises, chega-se à relação $y=10-\left(\frac{x}{2}-3,15\right)^{2}$. Foi possível observar, em um primeiro momento, um exercício de tentativas e erros para encontrar os parâmetros desejados, o que mostra a autonomia da ação no início deste processo. Contudo, W percebeu que essa estratégia não seria suficiente para obter êxito em suas ações; era preciso utilizar os conhecimentos matemáticos já construídos e aplicá-los nesta nova situação. A busca pelo estabelecimento destas novas relações evidencia a influência da conceituação sobre a ação, na medida em que $\mathrm{W}$ precisou utilizar o sistema de coordenadas cartesianas, analisar pontos tais como vértice, pontos de interseção com os eixos coordenados, para finalmente determinar a relação final de seu modelo matemático. Isso significa que a capacidade de previsão de W foi aumentando ao longo do processo de construção da relação matemática, evidenciando uma maior compreensão dos conceitos matemáticos relacionados com o problema.

Como o objetivo do trabalho era uma animação a ser desenvolvida no GeoGebra com a utilização do recurso "Controle Deslizante", a relação matemática deveria depender desta variável. Assim, um ponto A foi criado com as seguintes coordenadas: 
$\left(a, 10-(a / 2-3.15)^{2}\right)$, com $a$ variando entre 0 e 25 , obtendo o resultado gráfico ilustrado na Figura 2.

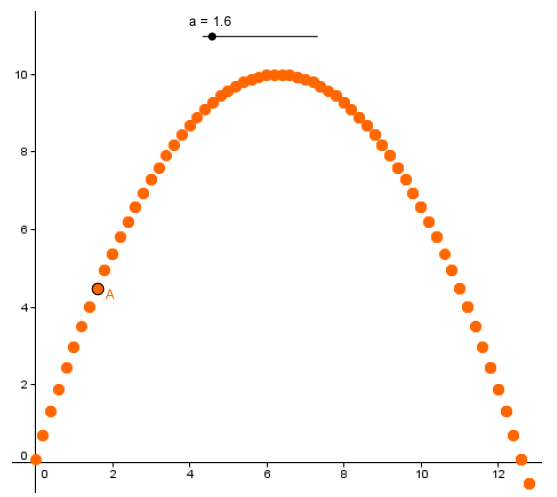

Figura 2. Primeira etapa do modelo - as coordenadas do ponto A

Dando continuidade à modelagem, $\mathrm{W}$ estabelece um novo desafio: modelar o efeito da trajetória da bola quando acerta a cesta. Para isso, foi preciso perceber que uma nova relação deveria ser introduzida ao modelo, para representar a bola caindo verticalmente até o chão. Estabelecer as posições do jogador e da cesta foram etapas importantes, para definir o instante em que a bola cai. Com estes elementos definidos, foi possível perceber que o ponto A estaria definido apenas para o intervalo $2<a<8$, pois, a partir de $a=8$, a bola deveria começar a cair verticalmente, obedecendo a uma nova relação. A Figura 3 ilustra esta etapa.

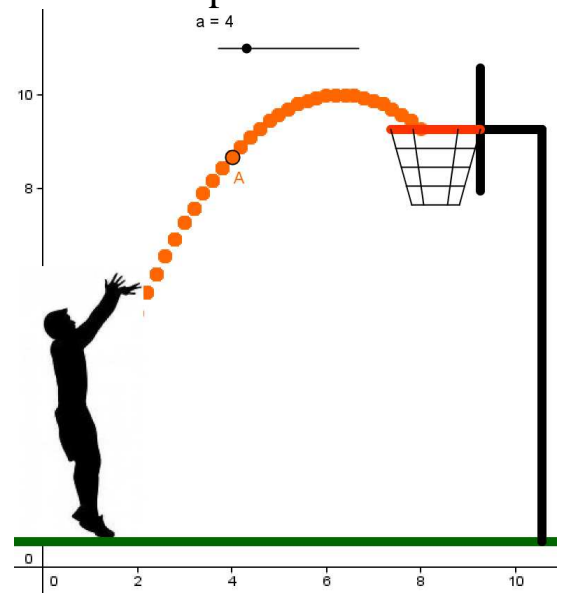

Figura 3. Segunda etapa do modelo - posições do jogador e da cesta

Encontrar as coordenadas do ponto A no momento exato para a troca de relação e estabelecer a nova relação, dando continuidade ao movimento, exigiram de $\mathrm{W}$ a compreensão de conceitos matemáticos importantes, como funções definidas por partes e continuidade de funções. Novamente, foi necessária uma capacidade de previsão de $\mathrm{W}$, o que aumentou sua capacidade de coordenação das ações que estavam sendo realizadas, levando a uma maior compreensão dos conceitos matemáticos utilizados. Percebe-se que o simples fazer já não basta; é necessário compreender o que se está fazendo, para estabelecer uma programação da ação a partir da conceituação.

Considerar que a variável $a$ já estava aumentando neste momento e estabelecer sua relação com a variável $x$ provocou um desequilíbrio em $\mathrm{W}$, pois a relação estabelecida inicialmente não desencadeou o efeito esperado. Neste momento, foi possível perceber, novamente, autonomia da ação e um atraso da conceituação, o que 
revela a necessidade de trilhar novamente o caminho do fazer ao compreender, agora nesta nova situação. Nesse caso, é possível perceber que a construção do conhecimento é um processo sem início nem fim, que estabelece a partir de idas e vindas, que vão continuamente reforçando e aumentado as estruturas cognitivas do sujeito. Após algumas tentativas e posteriores reflexões, $\mathrm{W}$ cria um ponto $\mathrm{E}$ de coordenadas $(8,17.3$ a), definido no intervalo $8 \leq a<16,5$.

Finalmente, no momento em que a bola toca o chão, W determinou uma continuidade em seu movimento, que desse a ideia de uma bola picando no chão. Novos conceitos matemáticos precisaram vir à tona, para definir uma relação matemática adequada e, mais do que isso, a continuidade do movimento. Um ponto $\mathrm{F}$ de coordenadas $(a-8,0.3+\operatorname{abs}(\sin (a))$, definido no intervalo $a>16,5$, foi criado para dar este efeito. A Figura 4 ilustra a trajetória completa da bola.

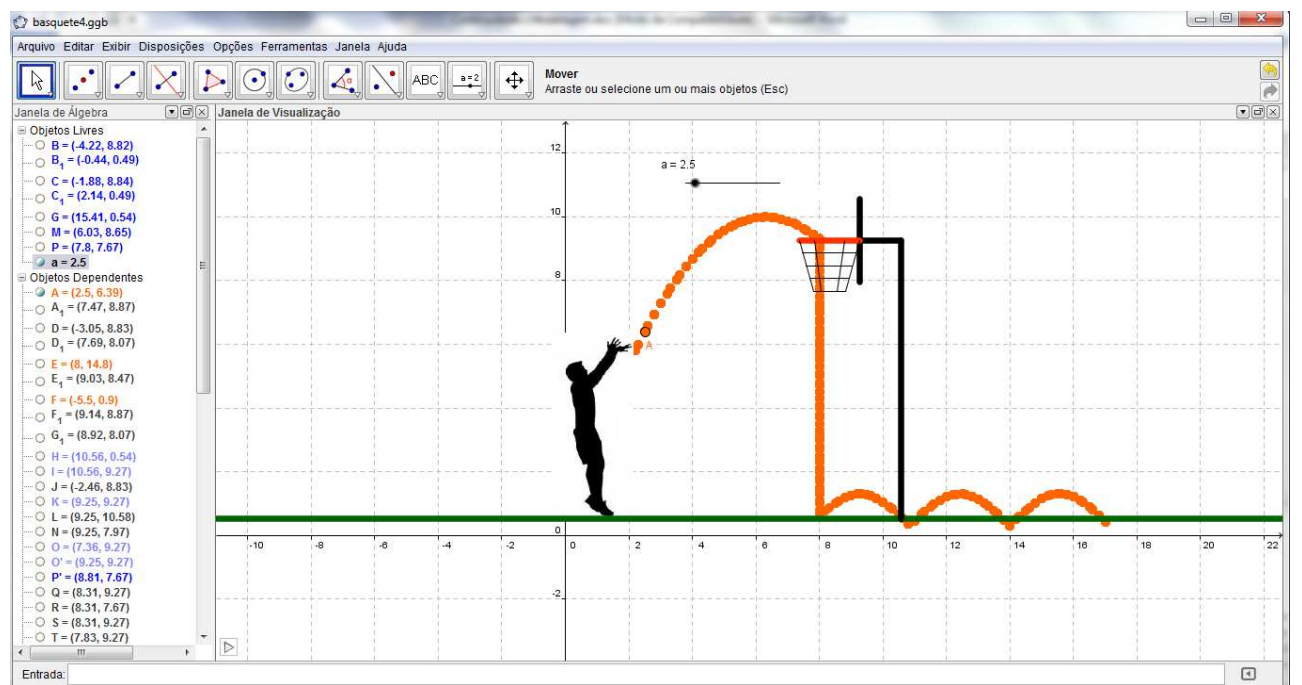

Figura 4. Trajetória da bola no modelo criado por W

Para finalizar seu trabalho, W associou a cada ponto a figura de uma bola de basquete, dando melhor acabamento ao seu modelo. A Figura 5 traz uma sequência de figuras que ilustram a modelagem de $\mathrm{W}$.
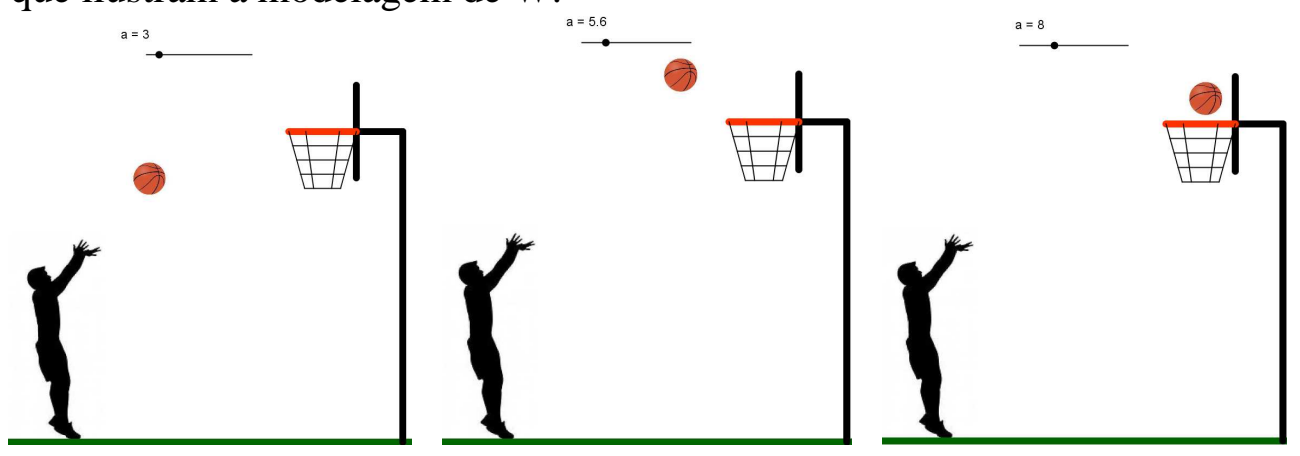


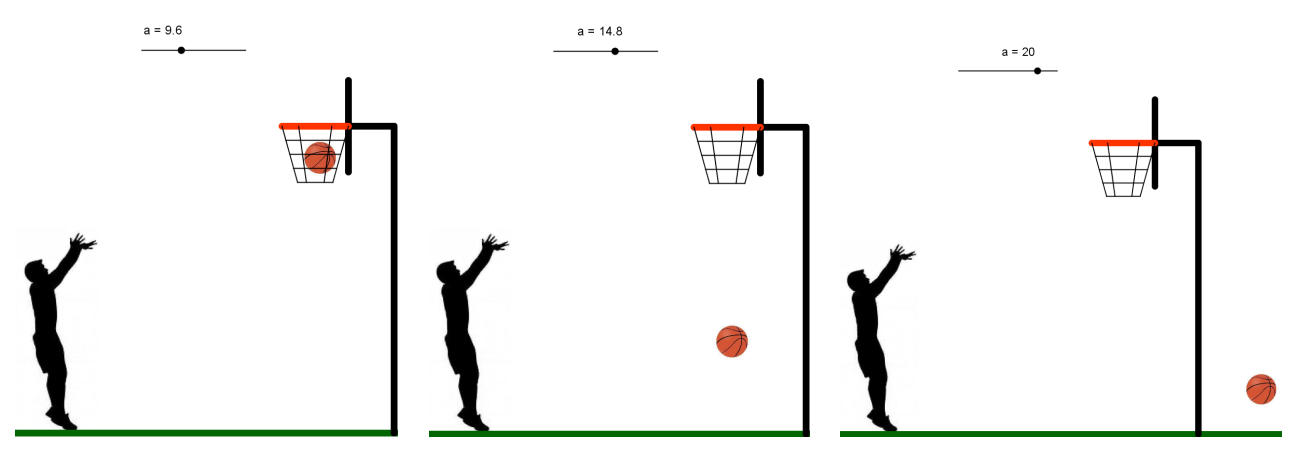

Figura 5. Imagens da animação do modelo de W

É claro que o modelo construído por W está longe da realidade, pois desconsiderou uma série de variáveis físicas que influenciam na trajetória da bola. Contudo, sob o ponto de vista da construção do conhecimento matemático, este trabalho de modelagem proporcionou um rico processo de tomada consciência de conceitos matemáticos, desencadeado pela necessidade de superar os obstáculos que surgiram ao longo da construção do modelo. Conceitos matemáticos importantes, como função quadrática, função afim, função seno, função valor absoluto, movimentos de gráficos (translação, reflexão e compressão) e continuidade de funções foram definitivos na construção do modelo. Ainda, conceitos geométricos como retas paralelas e perpendiculares, ponto médio, mediatriz e reflexão em torno de uma reta, foram utilizados na construção da cesta, para que se mantivesse estável sob ação do movimento. Dessa forma, a atividade proposta com o uso do GeoGebra permitiu a compreensão de uma série de conceitos a partir da ação do sujeito e da conceituação, trilhando um caminho do fazer ao compreender.

Assim, esse trabalho revela dois aspectos importantes: o potencial da tecnologia no processo de tomada de consciência de conceitos matemáticos e o potencial de uma teoria que sustenta a elaboração de atividades que possibilitem esse processo. Dessa forma, os alunos não aprendem Matemática por que alguém está ensinando; aprendem por um processo de construção e de tomada de consciência do próprio desenvolvimento. Mais do isso, os alunos realizam a atividade não para atender a exigência do professor, mas para atender suas necessidades individuais.

\section{Considerações Finais}

Este artigo buscou evidenciar o potencial da tecnologia no processo de construção do conhecimento matemático. À luz da teoria do desenvolvimento cognitivo de Piaget, buscou-se analisar o caminho trilhado por um aluno, do fazer ao compreender, em uma atividade de modelagem. Foi possível perceber que, quando engajado em uma atividade, o estudante pode atingir níveis mais elevados de compreensão de conceitos matemáticos, desencadeados pela necessidade de superar seu próprio desafio. $\mathrm{O}$ aluno W colocou-se frente a um desafio, de seu interesse, já que ele mesmo definiu o modelo a ser construído e, neste processo, construiu e deu novos significados a uma série de conceitos que nem mesmo ele previu que seriam necessários para a realização da atividade. Não havia um procedimento ou algoritmo a ser seguido, ou uma lista repetitiva de exercícios semelhantes; havia um desafio a ser superado. É com esse enfoque que defendemos a utilização da tecnologia nas aulas de Matemática: para proporcionar um rico espaço que permite a ação e a conceituação dos conceitos matemáticos, pois a construção do conhecimento matemático se dá na medida em que 
novos problemas matemáticos vão sendo vivenciados, uma vez que o aluno é perturbado e desafiado a superá-lo.

\section{Referências Bibliográficas}

DREYFUS, Tommy. Advanced Mathematical Thinking Processes. In: Tall, David (Ed.), Advanced Mathematical Thinking. Norwell: Kluver Academic Publishers, 1991, p. 25 - 40.

GRAVINA, M.; CONTIERO, L. Modelagem com o GeoGebra: uma possibilidade para a educação interdisciplinar? RENOTE: Revista Novas Tecnologias na Educação, Porto Alegre, v.9, n. 1, jul. 2011.

PIAGET, Jean. A Tomada de Consciência. São Paulo: Melhoramentos, Editora da Universidade de São Paulo, 1977.

PIAGET, Jean. Fazer e Compreender. São Paulo: Melhoramentos, Editora da Universidade de São Paulo, 1978.

PIAGET, Jean. A Epistemologia Genética. São Paulo: Abril Cultural, Coleção Os Pensadores, 1983.

PIAGET, Jean. Abstração Reflexionante. Porto Alegre: Artes Médicas, 1995.

TALL, David. The Psychology of Advanced Mathematical Thinking. In: Tall, David (Ed.), Advanced Mathematical Thinking. Norwell: Kluver Academic Publishers, 1991, p. $3-21$. 